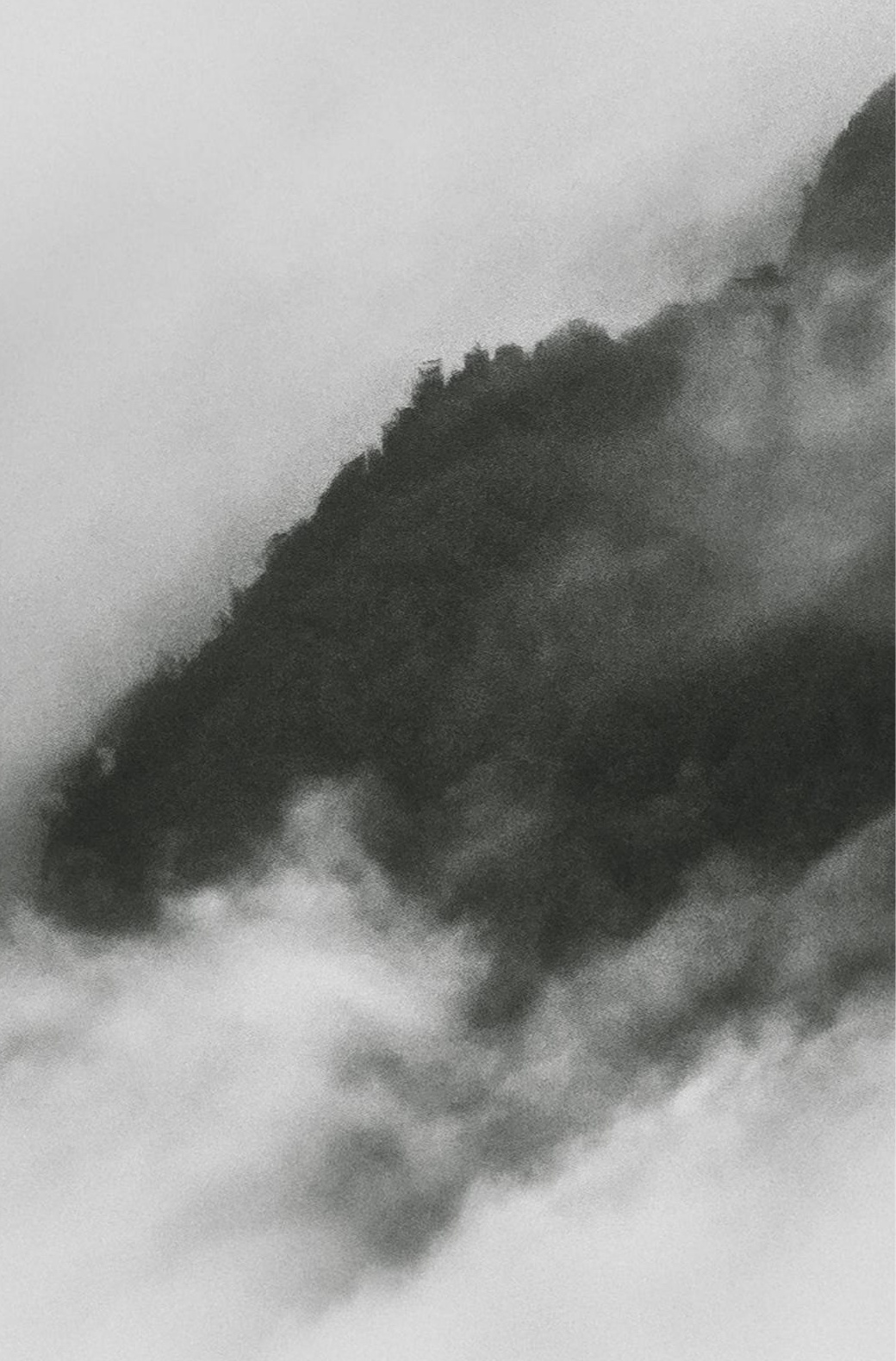




\section{Detrás de la neblina: lomas de Lima}

L

ima Metropolitana está rodeada de ecosistemas de lomas costeras. Sin embargo, son muy pocos los limeños que saben de su existencia - a pesar de que contamos con más de 21000 hectáreas de estas formaciones-, lo que ocasiona, entre otros factores, que se pierda y degrade el ecosistema. La población no ve lo que hay detrás de la neblina y se mantiene de espaldas a las lomas. Pero ¿cómo un recurso natural de tal magnitud, en una ciudad que demanda áreas verdes, pasa desapercibido para la mayoría? Algunos de los factores son el desconocimiento de la definición de lomas; la estacionalidad y dinámica del ecosistema, difícil de entender; la dinámica poblacional alrededor de ellas, y la desarticulación de los emprendimientos sociales locales para su defensa.

\section{Por Adriana Kato}


Lima, la gris. Así se le conoce a la capital del Perú debido a las nubes blancas y grisáceas que cubren su cielo durante el invierno e impiden el paso de los rayos solares por varios meses, lo cual ocasiona una alta humedad en la ciudad y molestias - como poca visibilidad, alergias, frío, afectación anímica, entre otros- en la mayoría de su población. Sin embargo, en una ciudad de contrastes como Lima, incluso el gris reverdece la capital, a través de un ecosistema único, especial y poco conocido como las lomas costeras.

Las lomas se encuentran a lo largo de toda la costa de Perú y Chile. En Lima Metropolitana, se extienden desde Ancón hasta Pucusana. Durante el invierno austral, el agua presente en la neblina se precipita sobre el suelo y permite que los bancos de semillas y bulbos de las especies adaptadas al desierto rebroten. Esta dinámica solo ocurre entre los meses de julio y octubre; sin embargo, algunas plantas de las lomas crecen todo el año debido a su nivel de adaptación, como bien indica el Programa de las Naciones Unidas para el Desarrollo (2018) - en adelante, PNUD-.

No obstante, a pesar de que Lima Metropolitana cuenta con más de 21000 hectáreas de lomas anuales (Proyecto EbA Lomas, 2017a), son muy pocos los ciudadanos limeños que conocen sobre su existencia y, menos aún, su importancia. Ello, junto a amenazas como el tráfico de terrenos, las invasiones, la minería ilegal y el sobrepastoreo, ocasiona el cambio de uso del suelo y dificultan su protección, conservación y gestión.

En este marco nace el proyecto "Conservación, gestión y rehabilitación de los ecosistemas frágiles de lomas en Lima", o EbA Lomas, llamado así por las siglas en inglés del enfoque que emplea: la adaptación basada en ecosistemas. Es implementado por el PNUD, bajo la dirección del Servicio Nacional de Áreas Naturales Protegidas por el Estado (Sernanp), órgano adscrito al Ministerio del Ambiente (Minam), con el financiamiento del Fondo para el Medio Ambiente Mundial (GEF).

El proyecto $\mathrm{EbA}$ Lomas tiene como objetivo proteger, conservar y restaurar los ecosistemas de lomas de Lima Metropolitana. Para alcanzar el objetivo, se elaboró una estrategia de comunicación, y como parte del diagnóstico, una de las principales preguntas que se buscó responder fue esta: ¿cómo un ecosistema que abarca gran parte de Lima es tan poco conocido por la población? En las siguientes páginas se presentan algunos de los factores que se detectaron y que dificultan la visibilización de las lomas y su comunicación.

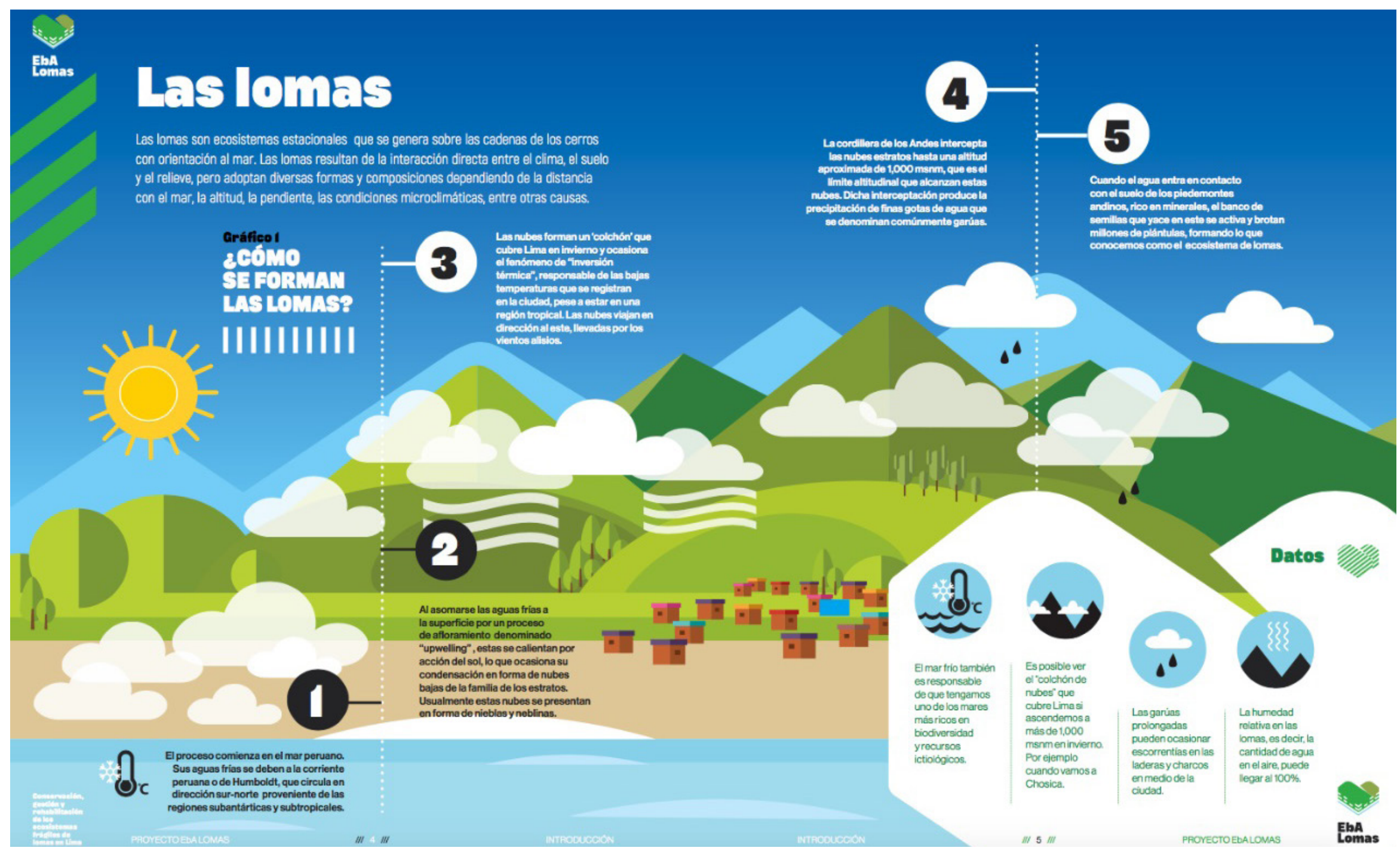

Figura 1. ¿Cómo se forman las lomas?

Fuente: Programa de las Naciones Unidas para el Desarrollo, 2018 


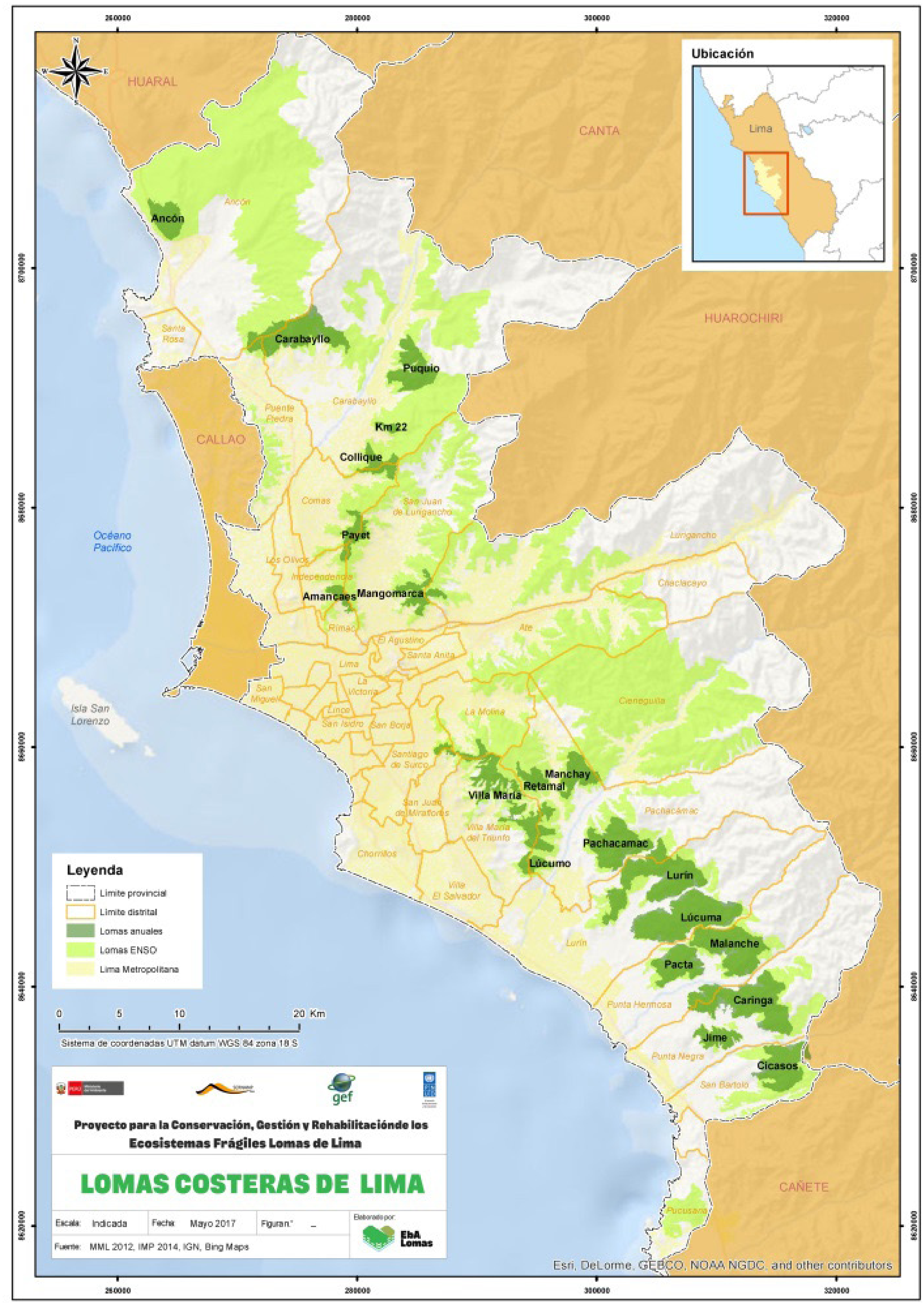

Figura 2. Mapa de las lomas de Lima Metropolitana Fuente: Proyecto EbA Lomas, 2017 


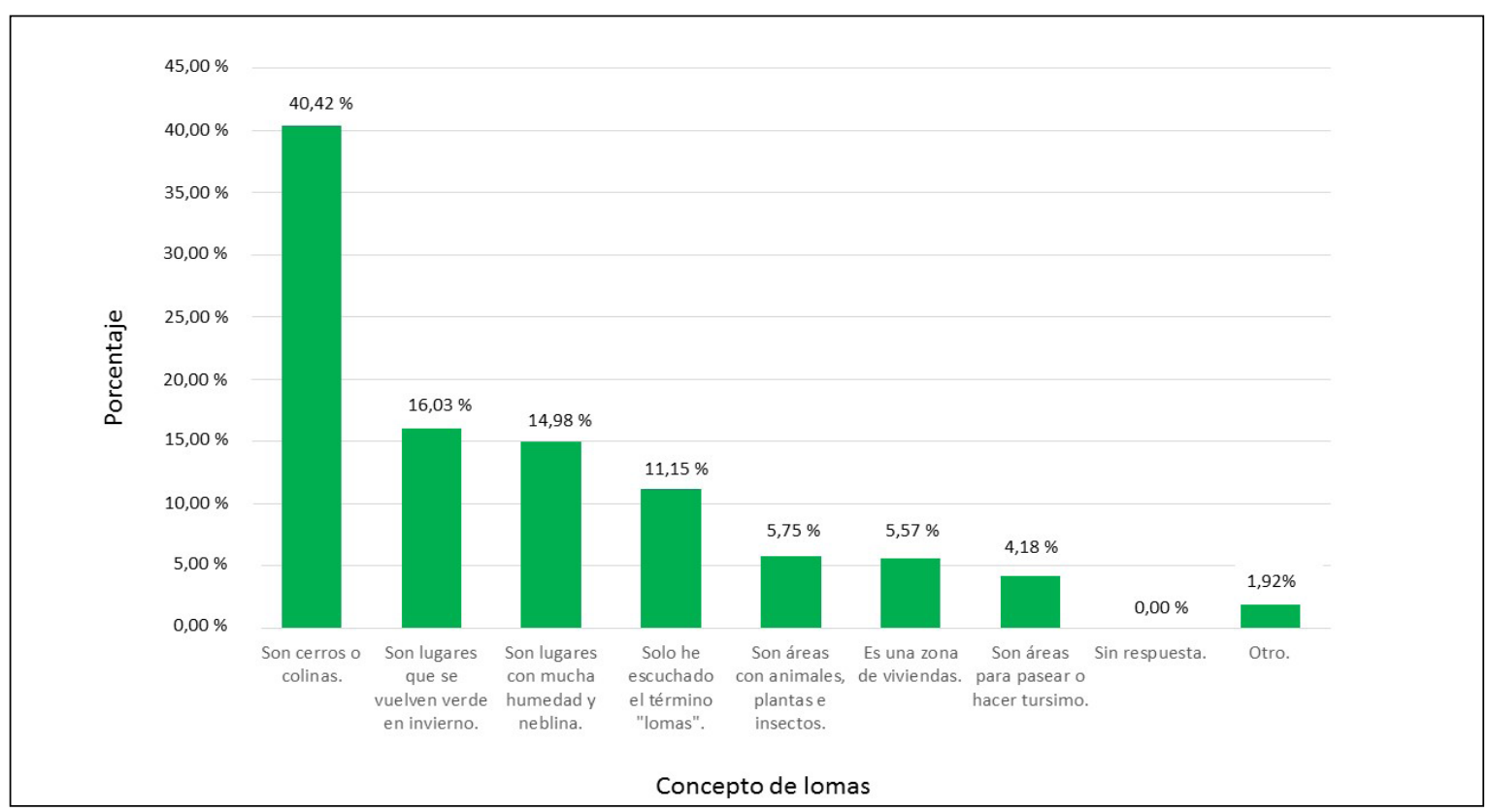

Figura 3. Concepto de lomas en Lima Metropolitana

Fuente: Instituto de Opinión Pública de la Pontificia Universidad Católica del Perú, 2017

\section{La definición y el concepto del término lomas}

Las lomas son ecosistemas que solo se encuentran en Perú y Chile, donde son conocidas como oasis de neblinas. El término loma, en la población en general, remite más al concepto geográfico de elevación, que predomina sobre el concepto ecológico. Incluso en el Diccionario de la lengua española de la Real Academia Española (RAE) solo encontramos esta definición:

\section{loma}

De lomo.

1. f. Altura pequeña y prolongada.

Como se ve, la etimología lingüística resulta confusa, pues el término loma proviene de lomo, del latín lomus, entendido como 'lomo' (anatomía). Desde la terminología solo se abarca una pequeña porción de lo que hoy entendemos como ecosistemas de lomas, y la mayoría de personas en Lima Metropolitana también la entiende de ese modo: como elevaciones, colinas o cerros, tal como muestra el Instituto de Opinión Pública de la Pontificia Universidad Católica del Perú (2017) -en adelante, IOP-PUCP-.

Una de las definiciones de lomas, desde la ecología, es la de "formaciones vegetales fuertemente estacionales y sustentadas por los frentes de neblinas invernales que se condensan en las primeras estribaciones andinas. [...] Se encuentran distribuidas en forma de islas continentales en medio de un "mar» de desierto" desde Trujillo, en Perú, hasta Coquimbo, en Chile (Arana y Salinas, 2007, p. 33).

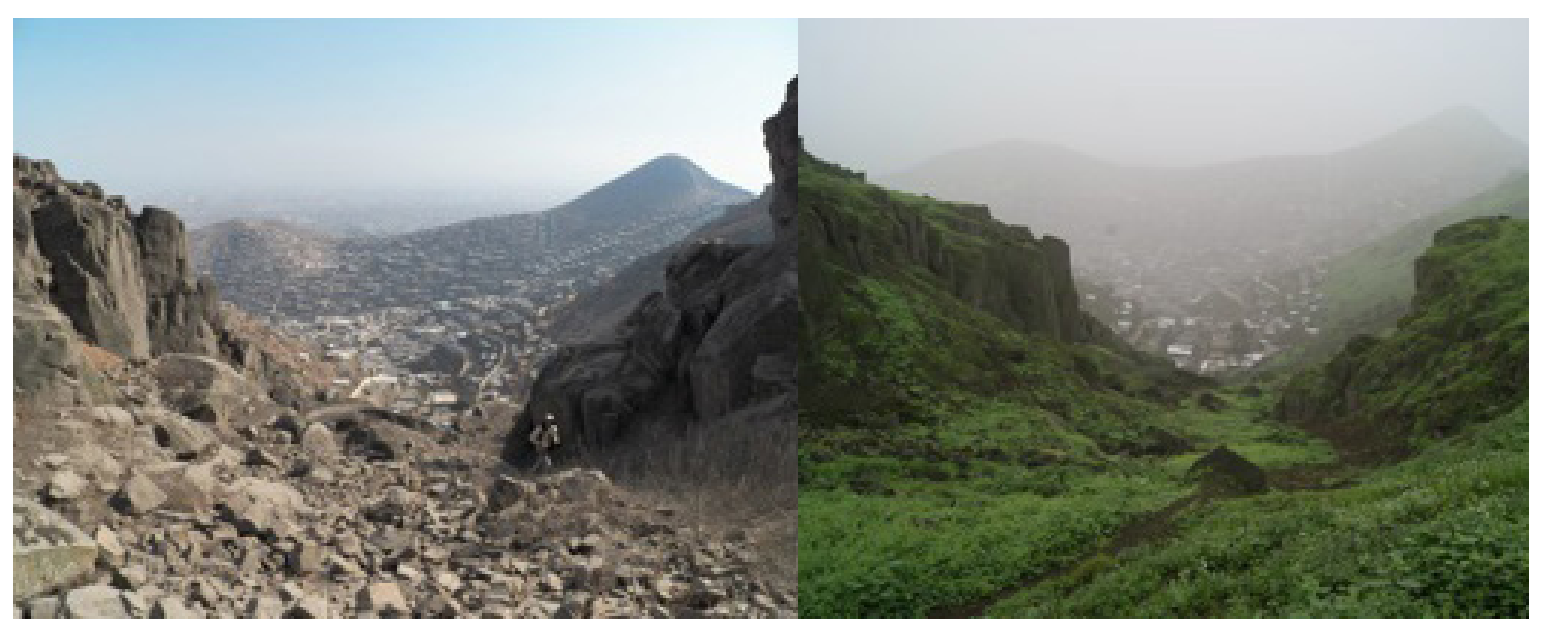

Figura 4. Lomas de Amancaes en temporada seca y en temporada húmeda Fuente: archivo fotográfico de Roobert Jiménez
Desde la comunicación, es necesario dar a conocer el concepto de ecosistemas de lomas y explicarlo en términos sencillos para que cualquier persona pueda entenderlo. Valdría la pena intentar acercarse a él a través de una definición simple, según las nociones que ya tienen las personas; por ejemplo, "cerros o colinas que se vuelven verde en invierno debido a la humedad y neblina”. De esta forma, así como un ciudadano tiene una noción medianamente clara de lo que es un bosque, un desierto o un pantano, lo mismo sucedería con las lomas.

\section{La estacionalidad y dinámica de las lomas: temporada seca y húmeda}

De por sí, ecosistemas no es un término común para el ciudadano de a pie. En la evaluación piloto del estudio de percepciones sobre las lomas de Lima (IOP-PUCP, 2017), la pregunta “ ¿Conoce o ha oído hablar sobre ecosistemas de lomas en Lima Metropolitana?" tuvo que ser modificada: se debió suprimir el término ecosistemas debido a que las personas no entendían el concepto, no lo relacionaban con lomas o se confundían.

$\mathrm{Si}$ a ecosistema se le agrega la palabra estacionalidad, se hace más difícil de entender aún. Lo cierto es que las lomas son ecosistemas estacionales: durante el invierno, las lomas reverdecen debido a que la neblina se precipita como garúa y genera una alta humedad en la ciudad; en el verano, la vegetación entra en reposo y solo unas cuantas especies florecen, como la flor trompeta (Stenomesson coccineum) (PNUD, 2018). Además, es un ecosistema dinámico, pues la composición de su biodiversidad y sus dimensiones varían de un año a otro con la presencia de un eventual incremento o disminución de humedad por los fenómenos climáticos de El Niño, La Niña y El Niño costero. 
Tabla 1. Conocimiento de la existencia de lomas cerca de la vivienda

\begin{tabular}{|lc|}
\hline \multicolumn{2}{|c|}{ ¿Ud. sabe si tiene una loma cerca de su vivienda? } \\
\hline & Total \\
No & $41,8 \%$ \\
Sí & $58,3 \%$ \\
Total (N) & 400 \\
\hline
\end{tabular}

Fuente: Instituto de Opinión Pública de la Pontificia Universidad Católica del Perú, 2017

A pesar de que es un ecosistema muy interesante, su visibilidad y comunicación respecto de la población genera un gran problema: en verano, cuando está despejado y claro, las lomas aparentan ser un cerro seco y pedregoso; y en invierno, cuando están verdes, en todo su esplendor y con mucha vegetación, están cubiertas por la típica neblina limeña.

Ello plantea un reto: comunicar la importancia de un ecosistema que no se ve y atraer a las personas. La mejor y, quizá, única forma de conocer lo que los ecosistemas de lomas ofrecen es experimentándolo, por lo que corresponde incentivar su visita para poder conservarlos.

\section{La dinámica poblacional cerca de las lomas}

A partir del estudio, se obtuvo una cifra impactante: la encuesta se aplicó en un radio aproximado de un kilómetro desde donde empiezan las lomas, y el 41,8 \% de los habitantes no sabía que tenía una loma cerca de su vivienda. ¿Cómo, en un radio tan limitado, no se aprecia tal recurso natural?

La explicación estaría en las actividades que realizan los habitantes de las zonas aledañas a las lomas. Sobre la expansión del uso residencial en laderas (muchas de ellas, laderas de las lomas), la Municipalidad Metropolitana de Lima (2014), diagnosticó lo siguiente:

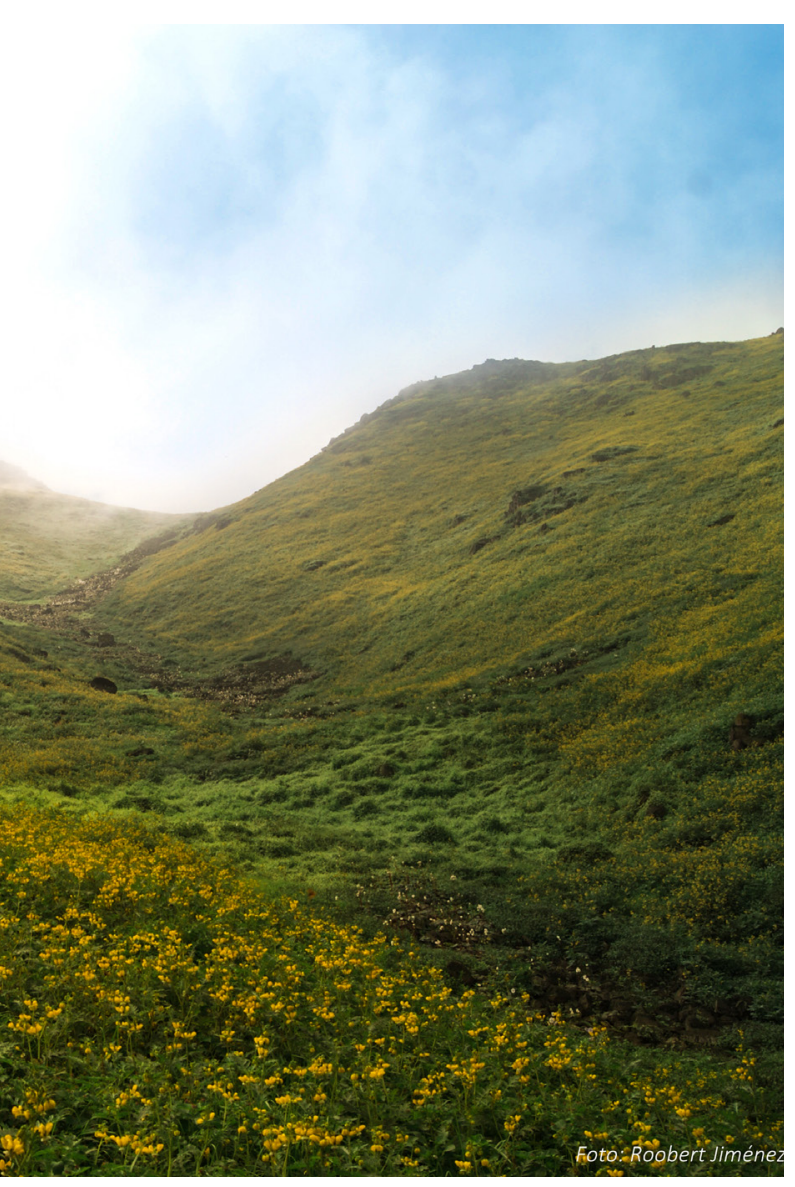

Además de corresponder a las últimas zonas de crecimiento urbano de la ciudad, tiene las condiciones de precariedad mayores por la falta de servicios básicos (agua y alcantarillado), material de construcción predominante no estructural y hacinamiento complementado con las limitaciones de accesibilidad. Son además exclusivas para uso de dormitorio de sus habitantes. [cursivas de la autora] (p. 389)

Los ecosistemas de lomas, al encontrarse en la periferia de Lima, albergan en sus zonas aledañas a habitantes que viven en "distritos dormitorios", es decir, un distrito que es utilizado principalmente para dormir, debido a que se trabaja o estudia en otro lugar. Entonces, no resulta raro que más del $40 \%$ de los habitantes no se haya percatado del recurso, pues muchos salen antes de que salga el sol y vuelven cuando ya ha oscurecido. De igual forma, como se mencionó, la estacionalidad de las lomas también dificulta su reconocimiento.

\section{Desarticulación \\ de emprendimientos sociales locales para la conservación y defensa de las lomas de Lima}

En Lima Metropolitana, hay una serie de emprendimientos sociales y locales de la sociedad civil, preocupados y comprometidos con la conservación de las lomas. Cada uno de estos emprendimientos trabaja arduamente por las lomas de su comunidad, ya sea tomando acciones legales o denunciando a quienes degradan o perjudican el ecosistema, planificando y realizando capacitaciones para el uso turístico de las lomas, buscando espacios para tener incidencia en tomadores de decisiones, u organizando campañas de reforestación y de limpieza.

Figura 5. Mangomarca

Fuente: archivo fotográfico de Roobert Jiménez 
Tabla 2. Emprendimientos sociales locales de la sociedad civil organizada para la conservación de las lomas en Lima Metropolitana

\begin{tabular}{|c|c|c|c|}
\hline Asociación local & Loma & Distrito & Actividad \\
\hline$\frac{\underline{\text { Asociación Circuito }}}{\text { Ecoturístico Lomas de }}$ & $\begin{array}{l}\text { Lomas } \\
\text { de Lúcumo }\end{array}$ & Pachacamac & $\begin{array}{l}\text { Gestores locales de las lomas de Lúcumo } \\
\text { en Quebrada Verde. Son los pioneros en la } \\
\text { puesta en valor de las lomas. Su emprendi- } \\
\text { miento social cuenta con más de veinte años } \\
\text { de experiencia. }\end{array}$ \\
\hline$\frac{\text { Asociación Ecoturística }}{\text { Lomas de Paraíso }}$ & Lomas de Paraíso & $\begin{array}{l}\text { Villa María } \\
\text { del Triunfo }\end{array}$ & $\begin{array}{l}\text { Asociación integrada por dirigentes locales } \\
\text { y jóvenes orientadores turísticos. Impulsan } \\
\text { la conservación de las lomas de Villa María } \\
\text { del Triunfo, el guiado turístico en el sector de } \\
\text { Paraíso, reforestaciones, limpiezas, etcétera. }\end{array}$ \\
\hline$\frac{\frac{\text { Comité Ecoturístico }}{\text { de las Lomas de }}}{\underline{\text { Mangomarca }}}$ & $\begin{array}{l}\text { Lomas de } \\
\text { Mangomarca }\end{array}$ & $\begin{array}{c}\text { San Juan } \\
\text { de Lurigancho }\end{array}$ & $\begin{array}{l}\text { Agrupación de vecinos que busca la } \\
\text { protección y conservación de las lomas de } \\
\text { Mangomarca. }\end{array}$ \\
\hline$\frac{\frac{\text { Asociación Ecológica }}{\text { Lomas de Primavera }}}{\underline{\text { Carabayllo }}}$ & $\begin{array}{l}\text { Lomas } \\
\text { de Primavera } \\
\text { Carabayllo }\end{array}$ & Carabayllo & $\begin{array}{l}\text { Emprendimiento social que busca la conser- } \\
\text { vación de las lomas de Carabayllo a través de } \\
\text { diversos mecanismos. Realiza actividades } \\
\text { de reforestación, limpieza, guiado turístico } \\
\text { y sensibilización en el sector de Primavera. }\end{array}$ \\
\hline$\frac{\underline{\text { Protectoras de la Flor y }}}{\text { las Lomas de Amancaes }}$ & $\begin{array}{c}\text { Lomas } \\
\text { de Amancaes }\end{array}$ & Rímac & $\begin{array}{l}\text { Organización local del Asentamiento } \\
\text { Humano Flor de Amancaes, conformada } \\
\text { por dirigentes y vecinos de siete sectores. } \\
\text { Han denunciado invasiones, acondicionado } \\
\text { senderos para el turismo, realizado guiados, } \\
\text { reforestaciones y otros. }\end{array}$ \\
\hline$\underline{\text { Haz tu Mundo Verde }}$ & $\begin{array}{l}\text { Lomas } \\
\text { de Amancaes, } \\
\text { Lomas } \\
\text { de El Mirador }\end{array}$ & $\begin{array}{c}\text { San Juan } \\
\text { de Lurigancho }\end{array}$ & $\begin{array}{l}\text { Organización juvenil que promueve la } \\
\text { conservación de las lomas y ofrece servicios } \\
\text { de deportes extremos como rappel, canopy, } \\
\text { trekking, etcétera. }\end{array}$ \\
\hline$\frac{\frac{\text { Instituto de Cultura, }}{\text { Historia y Medio }}}{\underline{\underline{\text { Ambiente }}}}$ & $\begin{array}{l}\text { Lomas de } \\
\text { Mangomarca }\end{array}$ & $\begin{array}{c}\text { San Juan } \\
\text { de Lurigancho }\end{array}$ & $\begin{array}{l}\text { Asociación civil sin fines de lucro confor- } \\
\text { mada por profesionales de San Juan de } \\
\text { Lurigancho, que trabaja en los ejes de educa- } \\
\text { ción, arte y cultura; patrimonio cultural y } \\
\text { natural; turismo y medioambiente. }\end{array}$ \\
\hline$\underline{\text { Kusi Sonqo }}$ & $\begin{array}{c}\text { Lomas de } \\
\text { Mangomarca }\end{array}$ & $\begin{array}{c}\text { San Juan } \\
\text { de Lurigancho }\end{array}$ & $\begin{array}{l}\text { Defensores del patrimonio cultural y } \\
\text { natural de San Juan de Lurigancho, de } \\
\text { la I. E. } 0090 \text { Daniel Alcides Carrión, de } \\
\text { Campoy. Guiados en el Ecocircuito Huaca } \\
\text { de Campoy y Lomas de Mangomarca. }\end{array}$ \\
\hline
\end{tabular}

Fuente: Proyecto EbA Lomas, 2017b

Elaboración propia 
Sin embargo, los emprendimientos sociales locales trabajan de forma independiente; es decir, cada uno trabaja de manera aislada, enfocándose en las lomas que están en sus distritos. Existen esfuerzos por agruparse, pero aún no se logra una organización sólida de los principales actores locales en las lomas de Lima Metropolitana, en la que puedan intercambiar experiencias y reforzarse entre ellos.

Todas las organizaciones son muy activas y buscan alianzas con diversas instituciones públicas o privadas. De lograr articular sus acciones y unir sus esfuerzos, se podría crear una agrupación con mucha fuerza, experiencia, pasión y notoriedad. Se podría pensar en una marca (Lomas de Lima, por ejemplo) que les permitiría recaudar fondos, establecer alianzas sólidas y posicionar a las lomas como símbolo de la ciudad.

\section{"Desde el Barranco a ver la flor de Amancaes"}

Las lomas son ecosistemas valiosos y en menos de diez años hemos perdido más de 1600 hectáreas de lomas (Proyecto EbA Lomas, 2017a). Si se conocieran más, habría más personas interesadas y preocupadas por preservarlas. Este es uno de los principales retos que tiene la comunicación de la mano con la educación: que la población local conozca y reconozca como propio el ecosistema de lomas.

Uno de los elementos clave con los que se podría llamar la atención de la población es a través de la flor de Amancaes (Ismene amancaes), que solo crece en las lomas. ¿Por qué no empezar por enseñarle a los niños y jóvenes cuál es la flor de Lima? ¿Por qué no recordarles lo que era la fiesta de San Juan de Amancaes en el Rímac? ¿Por qué no volver a cantar y hacer la ruta del "José Antonio" de Chabuca Granda? ¿Por qué no hablar de su inclusión en el logo de los Juegos Panamericanos Lima 2019?

Estos son solo algunos ejemplos de la forma en que las lomas podrían ponerse en valor a través de una estrategia de comunicación que involucre distintos actores - tanto a organizaciones de la sociedad civil y medios de comunicación como a los sectores privado y público-, con el fin de invitar a la población a que se una a los esfuerzos de conservación de este ecosistema único, pues

la pérdida de cualquiera de ellas [las lomas], o sus habitantes, sería un resultado muy triste para los esfuerzos globales de conservación. Este tipo de ecosistema no existe fuera de América del Sur, y no hay nada parecido en ningún otro lugar de la Tierra. (Dillon, 2015, p. 7)

\section{Referencias}

Arana, C., y Salinas, L. (2007). Fragilidad de los ecosistemas de lomas costeras del Perú central. Revista Científica Dilloniana, 5(1), 32-35.

Dillon, M. (2015). Presentación. En M. Lleellish, J. Odar y H. Trinidad, Guía de flora de las Lomas de Lima (p. 7). Lima: Servicio Nacional Forestal y de Fauna Silvestre.

Instituto de Opinión Pública de la Pontificia Universidad Católica del Perú. (2017). Estudio sobre percepciones de los residentes en las zonas periféricas a los ecosistemas de lomas y de los pobladores de la ciudad de Lima Metropolitana [documento interno elaborado por encargo del Proyecto EbA Lomas].

Loma. (2017). En Diccionario de la lengua española [versión en línea]. Recuperado el 13 de agosto del 2018 de http://dle. rae.es/?id=NaB7tlf

Municipalidad Metropolitana de Lima. (2014). Memoria de análisis y diagnóstico PLAM 2035 [documento interno].

Programa de las Naciones Unidas para el Desarrollo. (2018). Retos y oportunidades en la conservación de las lomas en Lima Metropolitana. Lima: Autor.
Proyecto EbA Lomas. (2017a). Estudio geográfico de las lomas de Lima Metropolitana [documento interno].

Proyecto EbA Lomas. (2017b). Mapeo de actores de las lomas

de Lima Metropolitana [documento interno].

\section{Bibliografía}

Lleellish, M., Odar, J., y Trinidad, H. (2015). Guía de flora de las lomas de Lima. Lima: Servicio Nacional Forestal y de Fauna Silvestre.

Servicio de Parques de Lima. (2014). Lomas de Lima. Futuros parques de la ciudad. Lima: Autor.

Valle, D. (2017). Informe línea base diversidad biológica. Lomas de Lima Sur. Lima: Programa de las Naciones Unidas para el Desarrollo [documento interno]. 